Pacific

Journal of

Mathematics

\title{
DISTANCE OF HEEGAARD SPLITTINGS OF KNOT COMPLEMENTS
}

MAGGY TOMOVA 


\title{
DISTANCE OF HEEGAARD SPLITTINGS OF KNOT COMPLEMENTS
}

\begin{abstract}
MAGgy TOMOVA
Let $K$ be a knot in a closed orientable irreducible 3-manifold $M$ and let $P$ be a Heegaard splitting of the knot complement of genus at least two. Suppose $Q$ is a bridge surface for $K$ and let $N(K)$ denote a regular neighborhood of $K$. Then either $d(P) \leq 2-\chi(Q-N(K))$, or $K$ can be isotoped to be disjoint from $Q$ so that after the isotopy $Q$ is a Heegaard surface for $M-N(K)$ that is isotopic to a possibly stabilized copy of $P$.
\end{abstract}

\section{Introduction}

A Heegaard splitting of a compact 3-manifold $M$ is a decomposition of the manifold into two compression bodies, $A$ and $B$. If the manifold is closed, $A$ and $B$ are handlebodies. The common boundary of $A$ and $B$ is called a Heegaard surface which we denote by $P$. We will write $M=A \cup_{P} B$.

The distance between any two essential simple closed curves $\alpha$ and $\beta$ in a Heegaard surface $P$ is the smallest integer $n \geq 0$ so there is a sequence of essential simple closed curves $\alpha=\alpha_{0}, \ldots, \alpha_{n}=\beta$ in $P$ such that for each $1 \leq i \leq n, \alpha_{i-1}$ and $\alpha_{i}$ can be isotoped to be disjoint in $P$. The distance of a Heegaard splitting $A \cup_{P} B, d(P)$, defined by Hempel [2001], is the smallest integer $n$ so that there is an essential curve $\alpha$ in $P$ which bounds a disk in $A$ and an essential curve $\beta$ which bounds a disk in $B$ and $d(\alpha, \beta)=n$. If $d(P)=0$ then we say that the Heegaard splitting $A \cup_{P} B$ is reducible, if $d(P)=1$ then we say that $A \cup_{P} B$ is weakly reducible and if $d(P) \geq 2$ then we say that $A \cup_{P} B$ is strongly irreducible.

Obtaining bounds on the distance of a Heegaard splitting $M=A \cup_{P} B$ is an interesting problem and much progress has been made recently. The first such bound is due to Hartshorn [2002], who used a closed essential surface in the manifold to give a bound on $d(P)$. This result was extended by Scharlemann to allow for possible boundary components of the essential surface.

Theorem 1.1 [Scharlemann 2006, Theorem 3.1]. Suppose P is a Heegaard surface for a compact orientable manifold $M$ and $(Q, \partial Q) \subset(M, \partial M)$ is a connected essential surface. Then $d(P) \leq 2-\chi(Q)$.

MSC2000: 57M25, 57M27, 57M50.

Keywords: knot distance, bridge surface, Heegaard splitting.

This works was partially supported by NSF grant DMS-0704207. 
Here is a bound on $d(P)$ using a second Heegaard surface:

Theorem 1.2 [Scharlemann and Tomova 2006a, Corollary 3.5]. Suppose P and $Q$ are distinct Heegaard splittings for the compact orientable 3-manifold $M$. Then either $d(P) \leq 2-\chi(Q)$ or $Q$ is isotopic to a stabilization of $P$.

Suppose a manifold $M$ contains a knot $K$ and let $M_{K}$ be the knot complement. We say that a surface $Q$ is a bridge surface for $K$ if $Q$ is a Heegaard surface for $M=X \cup_{Q} Y$ and $K$ intersects each of the compression bodies $X$ and $Y$ in arcs that are simultaneously parallel to $Q$. In this paper we obtain a bound on the distance of Heegaard splittings of $M_{K}$ using a bridge surface for the knot.

Johnson and Thompson [2006, Theorem 1] used Theorem 1.2 to show that if a knot $K \subset M$ with bridge surface $Q$ is such that $K$ cannot be isotoped to lie in $Q$, then the distance of any Heegaard splitting of the knot complement is bounded by $\chi(Q-N(K))$ where $N(K)$ is a regular neighborhood of $K$. They used this theorem to obtain tunnel number one knots that have arbitrarily high bridge number with respect to any bridge surfaces of genus 1. Kobayashi and Rieck [2007, Proposition 2.6] extended this result to obtain the same bound on the distance of a Heegaard splitting in the case when the genus of the bridge surface $Q$ is strictly less than the minimum genus of any Heegaard splitting of the knot complement. Their result allows for the knot to be isotopic to an embedded curve in $Q$. They used this distance bound to prove the existence of counterexamples to Morimoto's conjecture [2000, Conjecture 1.5].

In this paper we remove the restriction on the genus of $Q$ as well as restrictions on whether the knot is isotopic into $Q$. More precisely:

Theorem 1.3. Let $K$ be a knot in a closed oriented irreducible 3-manifold $M$ and let $P$ be a Heegaard splitting of the knot complement of genus at least two. Suppose $Q$ is a bridge surface for $K$ and let $N(K)$ denote a regular neighborhood of $K$. Then either

- $d(P) \leq 2-\chi(Q-N(K))$, or

- $K$ can be isotoped off $Q$ so that after the isotopy $Q$ is a Heegaard surface for the knot complement, isotopic to a possibly stabilized copy of $P$.

In some sense this theorem completes the solution to the problem as it is clear that a stabilized copy of $P$ will not carry any information about $d(P)$ and these are the only bridge surfaces which we exclude. This result was used by Minsky, Moriah and Schleimer [Minsky et al. 2006] to show that for any pair of integers $t$ and $b$ there is a knot with tunnel number $t$ such that every bridge surface of genus $t$ for the knot intersects the knot in at least $2 b+2$ points. The proof of Theorem 1.3 uses different methods than those used in [Johnson and Thompson 2006] and [Kobayashi and Rieck 2007] and is independent of them. 


\section{Definitions and notation}

In this paper, unless otherwise specified, we will consider a closed irreducible orientable 3-manifold $M$ containing a knot $K$. If $X$ is any subset of $M$ we will denote by $X_{K}$ the set $X-N(K)$, where $N(K)$ is an open tubular neighborhood of $K$. In particular the knot exterior $M-N(K)$ will be denoted by $M_{K}$. Note that $M_{K}$ is a compact orientable manifold with a single torus boundary component. Throughout this paper $P$ will be a surface splitting $M$ into handlebodies $A$ and $B$ so that $K$ is entirely contained in $A$. We will further assume that $A_{K}$ is a compression body, that is $P$ is also a Heegaard surface for $M_{K}$ and $M_{K}=A_{K} \cup_{P} B$. We will further assume that $P$ has genus at least 2 as otherwise distance is always infinite with our definition.

Recall that a simple closed curve in a compact surface $S$ is essential if it does not bound a disk in the surface and it is not parallel to a boundary component of the surface. Suppose $M$ is a closed manifold containing a knot $K$ and $S$ is a surface in $M$ transverse to $K$. A disk $D \subset M_{K}$ is a compressing disk for $S_{K}$ if $D \cap S=\partial D$ and $\partial D$ is an essential simple closed curve in $S_{K}$. A disk $D^{c}$ in $M$ is a cut disk for $S_{K}$ if $D^{c} \cap S_{K}=\partial D^{c}, \partial D^{c}$ is essential in $S_{K}$ and $D^{c}$ intersects $K$ in a single point. A $c$-disk is a cut or a compressing disk.

A properly embedded surface $F$ in a 3-manifold $M$ is essential if it is incompressible and has at least one component that is not parallel to $\partial M$.

A surface $F$ in $M$ is called a splitting surface if $M$ can be written as the union of two 3-manifolds $U$ and $V$ along $F$. If $F$ is a splitting surface it is bicompressible if it is compressible in both $U$ and $V$ and $F$ is c-bicompressible if it has c-disks in both $U$ and $V$. If $F$ is a splitting surface for $M$, we will call $F c$-weakly incompressible if it is c-bicompressible and any pair of c-disks for $F$ on opposite sides of the surface intersect along their boundaries. If a c-bicompressible surface $F$ is not c-weakly incompressible, it is c-strongly compressible.

Suppose $K$ is a knot in a closed orientable 3-manifold $M$. We say that a surface $Q$ is a bridge surface for $K$ if $Q$ is a Heegaard surface for $M=X \cup_{Q} Y$ and $K$ intersects each of the handlebodies $X$ and $Y$ in arcs that are simultaneously parallel to $Q$. More generally, if the manifold $M$ has boundary, we require that $K$ intersect each of the compression bodies $X$ and $Y$ in arcs that are simultaneously parallel into $Q$. The arcs are called bridges and the disks of parallelism are called bridge disks. A handlebody $H$ intersecting a knot $K$ in a collection of bridges will be called a $K$-handlebody and will be denoted by $(H, K)$.

A spine $\Sigma_{H}$ of a handlebody $H$ is any graph that $H$ retracts to. Removing a neighborhood of a spine from a handlebody results in a manifold that is homeomorphic to surface $\times I$. More generally the spine $\Sigma_{C}$ of a compression body $C$ is the union of $\partial_{-} C$ and a 1-complex $\Gamma$ so that $C$ collapses to $\Sigma_{C}$. 
Suppose $(H, K)$ is a $K$-handlebody and let $\kappa_{i}, i=1, \ldots, n$ be the bridges. The spine $\Sigma_{(H, K)}$ of $(H, K)$ is the union of a spine of the handlebody $H, \Sigma_{H}$, together with a collection of straight arcs $t_{i}$ where one endpoint of each $t_{i}$ lies in $\kappa_{i}$ and the other endpoint lies in $\Sigma_{H}$. Note that $H_{K}-\Sigma_{(H, K)} \cong(\partial H)_{K} \times I$. As in the handlebody case, spines of $K$-handlebodies are not unique.

A bridge surface $Q$ for $K$ is called stabilized if there is a pair of compressing disks for $Q$, one in $X$ and one in $Y$, that intersect in exactly one point. The surface $Q$ is called meridionally stabilized if there is a compressing disk for $Q$ in $X$ and a cut disk for $Q$ in $Y$ (or vice versa) that intersect in exactly one point. Finally $Q$ is called perturbed if there is a pair of bridge disks, $E_{X} \subset X$ and $E_{Y} \subset Y$, such that $E_{X} \cap E_{Y}=p$ and $p \in K$. By [Scharlemann and Tomova 2006b], if $Q$ is stabilized, meridionally stabilized or perturbed, there is a bridge surfaces $Q^{\prime}$ for $K$ such that $\chi\left(Q_{K}^{\prime}\right)>\chi\left(Q_{K}\right)$. Furthermore it is easy to see that if $Q$ is stabilized, meridionally stabilized or perturbed then it is c-strongly compressible, see [Tomova 2007, Proposition 7.4] for the stabilized and meridionally stabilized case.

Suppose $Q$ is a bridge surface for a knot $K \subset M$. We say that $K$ is removable with respect to $Q$ if it can be isotoped to lie in $Q$ and there is a meridian disk for one of the two handlebodies $X$ and $Y$ that intersects $K \subset Q$ in a single point. In this case, by Lemma 3.3 in [Scharlemann and Tomova 2006c], there is a Heegaard splitting $\tilde{Q}$ for $M_{K}$ such that $\chi\left(\tilde{Q}_{K}\right)>\chi\left(Q_{K}\right)$.

We will also need the following result, shown in [Tomova 2006].

Theorem 2.1. Suppose $M$ is a closed orientable irreducible 3-manifold containing a knot $K$. If $Q$ is a c-strongly compressible bridge surface for $K$ then one of the following is satisfied:

- $Q$ is stabilized.

- $Q$ is meridionally stabilized.

- $Q$ is perturbed.

- $K$ is removable.

- $M$ contains an essential meridional surface $F$ such that

$$
2-\chi\left(F_{K}\right) \leq 2-\chi\left(Q_{K}\right) .
$$

\section{Preliminary results}

Recall that we are considering a Heegaard splitting $A_{K} \cup_{P} B$ of a knot complement $M_{K}$ where $M$ is a closed manifold. How surfaces in a manifold restrict the distance of a Heegaard splitting in various settings has been studied in several papers, including [Bachman and Schleimer 2005; Scharlemann and Tomova 2006a; Tomova 2007]. We will take advantage of some of these results. 
Proposition 3.1 [Scharlemann 2006, Proposition 2.5]. Let $M$ be an irreducible 3manifold, $N$ a compressible boundary component of $M$ and $(F, \partial F) \subset(M, \partial M)$ a properly embedded essential surface containing no disk components and with at least one essential component incident to $N$. Let $\mathscr{V}$ be the set of essential curves in $N$ that bound disks in $M$ and let $f$ be any component of $\partial F$. Then either

$$
d(\mathscr{V}, f) \leq 1-\chi(F)
$$

or $f$ lies in the boundary of $\partial$-parallel annulus component of $F$.

We will use the proposition above in a very specific situation. The manifold $M$ will either be the handlebody $B$ or the compression body $A_{K}$ and $N$ will be $\partial_{+} A_{K}=\partial B=P$. Then the above proposition says roughly that the distance between the boundary curves of an essential surface in a compression body and the boundaries of the compressing disks for the compression body is bounded above by the Euler characteristic of the essential surface.

The situation becomes considerably more complicated if we allow the surface $F$ to have compressing disks. However, if we restrict our attention to weakly incompressible surfaces, useful information about $d(\mathscr{V}, f)$ can still be obtained; see [Scharlemann 2006; Tomova 2007]. First we recall the definition of a tubespanned recessed collar. Let $S_{0}, S_{1}$ be two connected compact subsurfaces in the same component $N$ of $\partial M$, with each component $\partial S_{i}, i=0,1$, essential in $\partial M$ and $S_{0} \subset$ interior $\left(S_{1}\right)$. Let $T_{i}, i=0,1$ be the properly embedded surface in $M$ obtained by pushing $S_{i}$, rel $\partial S_{i}$ into the interior of $M$, so the region $R$ lying between $T_{0}$ and $T_{1}$ is naturally homeomorphic to $S_{1} \times I$. The properly embedded surface obtained by tubing $T_{0}$ and $T_{1}$ along an $I$-fiber of $S_{1} \times I$ that is incident to $T_{0}$ is called a tube-spanned recessed collar in $M$. The properties of these surfaces are described in detail in [Scharlemann 2006]. It turns out that tube spanned recessed collars are the only weakly incompressible surfaces that don't carry information about distance. More precisely:

Theorem 3.2 [Scharlemann 2006, Theorem 5.4]. Suppose $M$ is an irreducible 3manifold, $N$ is a compressible boundary component of $M$ and $(F, \partial F) \subset(M, \partial M)$ is a bicompressible, weakly incompressible splitting surface with a bicompressible component incident to $N$.

Let $\mathscr{V}$ be the set of essential curves in $N$ that bound disks in $M$ and let $f$ be any component of $\partial F \cap N$. Then either

- $d(\mathscr{V}, f) \leq 1-\chi(F)$, or

- $f$ lies in the boundary of d-parallel annulus component of $F$, or

- one component of $F$ is a tube spanned recessed collar; all other components of $F$ incident to $N$ are incompressible and d-parallel. 
Again, we will only be considering the situation when $M$ is the handlebody $B$ or the compression body $A_{K}$. Note that in these cases, if the surface $F$ consists of a single component which is a tube-spanned recessed collar with boundary in $\partial_{+} A_{K}$ or in $\partial B$, then there is a spine for $A_{K}$ or $B$ that is entirely disjoint from $F$ and $F$ has a compressing disk (a meridional disk for the tube) that lies on the same side of $F$ as the spine and is disjoint from the spine.

The above two results tell us that if there is a surface with certain properties in a compression body, then the boundary curves are "not far" from the boundaries of the compressing disks for the compression body. Thus if $U \cup_{R} V$ is a Heegaard splitting for a manifold, $\mathcal{U}$ and $\mathscr{V}$ are the collections of simple closed curves in $R$ that bound disks on sides $U$ and $V$ respectively and $S$ is a surface that intersects $R$ in a particular way, then we can hope to obtain a bound on the distance of $R$ by summing $d(\mathcal{U}, R \cap S)$ and $d(R \cap S, \mathscr{V})$. The next lemma makes this idea precise.

Lemma 3.3 [Scharlemann and Tomova 2006a, Lemma 2.6]. Suppose R is a Heegaard splitting for a compact manifold $M$, dividing $M$ into compression bodies $U$ and $V$. Let $U$ and $\mathscr{V}$ be the collections of simple closed curves in $R$ that bound disks in sides $U$ and $V$ respectively. Let $S \subset M$ be a properly embedded connected surface transverse to $R$, let $S^{U}=S \cap U$ and let $S^{V}=S \cap V$. Suppose that

- all curves of $S \cap R$ are essential in $S$ and in $R$;

- there is at least one curve $u \in S \cap R$ such that $d(u, \mathcal{U}) \leq 1-\chi\left(S^{U}\right)$ and any curve in $S \cap R$ for which the inequality does not hold is the boundary of an annulus component of $S^{U}$ that is parallel into $R$;

- there is at least one curve $v \in S \cap R$ such that $d(v, \mathscr{V}) \leq 1-\chi\left(S^{V}\right)$ and any curve in $S \cap R$ for which the inequality does not hold is the boundary of an annulus component of $S^{V}$ that is parallel into $R$.

Then $d(R) \leq 2-\chi(S)$.

The following lemma is similar to Lemma 3.6 in [Tomova 2007] where the result was proven in the context of a $K$-handlebody. We will need the result for a compression body so some modifications in the proof are needed.

Lemma 3.4. Let $M$ be a closed orientable irreducible 3-manifold containing a knot $K$. Suppose $P$ and $Q$ are Heegaard surfaces for $M=A \cup_{P} B=X \cup_{Q} Y$. In addition, suppose that $P$ is also a Heegaard surface for $M_{K}=A_{K} \cup_{P} B$ and $Q$ is a bridge surface for $K$. If there is a spine $\Sigma_{B}$ for $B$ lying in $Y$ and $Q_{K}$ has a c-disk $D^{*}$ in $Y_{K}-P$ that is disjoint from $\Sigma_{B}$ then either

- $Q$ is c-strongly compressible, or

- $K$ is removable with respect to $Q$, or

- $M=S^{3}$ and $K$ is the unknot. 
Proof. As already noted, $B-\Sigma_{B}$ has a natural product structure. Use this structure to push $Q_{K}$ and $D^{*}$ to lie entirely in $A_{K}$. As $X \subset A_{K}, Q_{K}$ always compresses in $X \cap A_{K}$ and thus we may assume that $\left(Q_{K}, \partial Q_{K}\right) \subset\left(A_{K}, \partial A_{K}\right)$ is a c-weakly incompressible surface.

Case 1: Suppose first that $D^{*}$ is a disk. In this case $Q_{K}$ is a weakly incompressible surface lying in the compression body $A_{K}$. Maximally compress $Q_{K}$ in $Y_{K} \cap A_{K}$ and let $Q_{K}^{\prime}$ be the resulting surface. Note that by the construction, $Q_{K}^{\prime}$ separates $P$ and $Q_{K}$. It is a classical result that maximally compressing a weakly incompressible surface results in an incompressible surface, see for example [Scharlemann 2006, Lemma 5.5]. As $Q_{K}^{\prime}$ is an incompressible surface in a compression body and $\partial Q_{K} \subset \partial_{-} A_{K}$, each component of $Q_{K}^{\prime}$ must be parallel to $\partial A_{K}$, that is, each component is an annulus or a torus. As $Q_{K}^{\prime}$ separates $P$ and $Q_{K}$ some component $Q_{K}^{0}$ of $Q_{K}^{\prime}$ also separates $Q_{K}$ and $P$.

Subcase 1A: Suppose first that $Q_{K}^{0}$ is an annulus. Then the corresponding closed component $Q^{0}$ of $Q^{\prime}$ bounds a ball in $A$ containing $Q$. As $Q_{K}^{\prime}$ is parallel to $\operatorname{Fr}(N(K)), K$ intersects this ball in a trivial arc. Now consider $Q_{K}^{0}$ as a surface in the $K$-handlebody $Y$. As handlebodies are irreducible, $Q_{K}^{0}$ must also bound a ball in $Y$ and by Lemma 3.2 of [Scharlemann and Tomova 2006b], the knot intersects this ball in a trivial arc. Thus $M$ is the three sphere and $K$ is a one bridge knot with respect to the bridge sphere $Q^{0}$, thus $K$ is the unknot.

Subcase $1 B$ : Suppose then that $Q^{0}$ is a torus bounding a solid torus $V$ in $A$ which is a regular neighborhood of $K$. As $Q_{K}^{\prime}$ is obtained from $Q_{K}$ by compressing along a collection of disks, the original surface $Q_{K}$ can be recovered from $Q_{K}^{\prime}$ by tubing along a graph $\Gamma$ with edges dual to these disks.

If $g(Q)=1, Q^{0}$ is the only torus component of $Q_{K}^{\prime}$ and all edges of $\Gamma$ have endpoints on two different components of $Q_{K}^{\prime}$. Pick a meridional disk $F$ for $Q^{0}$ that intersect $K$ exactly once. Isotope $K$ to lie in $Q^{0}$ using the parallelism of $Q^{0}$ and $\operatorname{Fr}(N(K))$, this isotopy is not proper. After the isotopy of $K$ each edge of $\Gamma$ has at least one endpoint on a sphere that bounds a ball in $V$. By shrinking $\Gamma$ we can guarantee that the graph is disjoint from $F$. Thus $F$ is also a compressing disk for $Q$ that intersects $K$ in a single point along its boundary, i.e., $K$ is removable with respect to $Q$.

If $g(Q)>1$, consider the two components of $V-Q_{K}$. One component is $X_{K}$ and so it is a handlebody intersecting the knot in trivial arcs. The other component can be obtained by attaching 1-handles corresponding to the edges of $\Gamma$ to a collar of $Q_{K}^{\prime}$. The result is a compression body $Y^{\prime}$ which intersects $K$ in arcs that are parallel into $\partial_{+} Y^{\prime}$. Thus $X \cup_{Q} Y^{\prime}$ is a bridge surface for $K$ lying in the manifold $V$. As $g\left(Q_{K}\right) \geq 2$, by [Hayashi and Shimokawa 2001] this implies that $X \cup_{Q} Y^{\prime}$ is stabilized or perturbed. Thus $Q_{K}$ is c-strongly compressible in $M$ as desired. 
Case 2: Suppose now that $D^{*}$ is a cut disk. As $A_{K}$ is a compression body, there is a spanning annulus $\Lambda$ in $A$ with one boundary component in $\operatorname{Fr}(N(K))$ and the other in $P$. As $Q \cap K \neq \varnothing$, it follows that $Q \cap \Lambda \neq \varnothing$ and in particular $Q$ intersects $\Lambda$ in at least one arc. As $Q \cap P=\varnothing$ any such arc must have both of its endpoints in $K$. As $D^{*}$ is a cut disk there is an $\operatorname{arc} \gamma$ in $D^{*} \cap \Lambda$ with one boundary component in $\operatorname{Fr}(N(K))$ and the other in some component of $Q \cap \Lambda$, as in the figure:

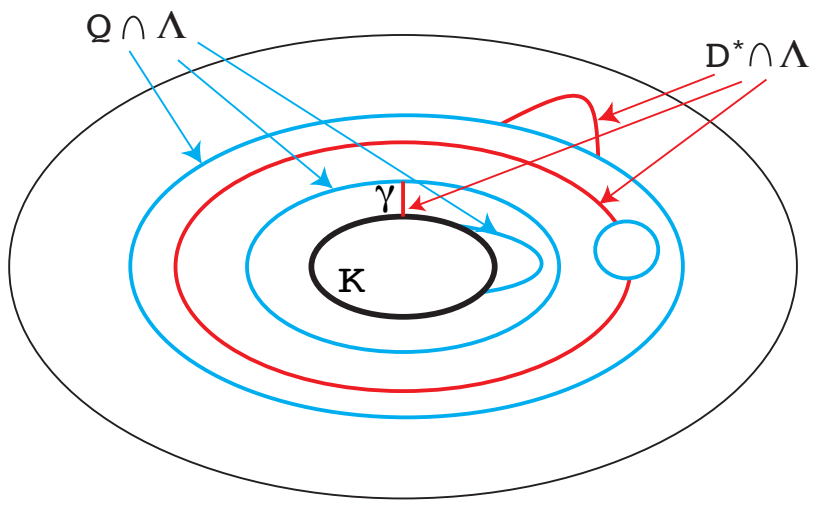

Choose $\Lambda$ and $D^{*}$ such that the tuple $\left(\left|D^{*} \cap \Lambda\right|,|Q \cap \Lambda|\right)$ is minimal in the lexicographic order.

Claim 1. $D^{*} \cap \Lambda$ does not contain any simple closed curves.

Suppose $\sigma$ is a simple closed curve in $D^{*} \cap \Lambda$ that is innermost in $D^{*}$. As $\sigma$ is disjoint from $\gamma$, the disk $E \sigma$ bounds in $D^{*}$ is disjoint from $K$. If $\sigma$ is inessential in $\Lambda$ then replace the disk $\sigma$ bounds on $\Lambda$ with $E$ reducing $D^{*} \cap \Lambda$. If $\sigma$ is parallel to the core of $\Lambda$, then $\sigma \cup K$ cobound a subannulus of $\Lambda$. The union of this subannulus and $E$ is then a compressing disk for $\partial_{-} A_{K}$. As the negative boundary of a compression body is incompressible, we have reached a contradiction.

Claim 2. $D^{*} \cap \Lambda$ does not contain any arcs with both endpoints in $Q \cap \Lambda$.

Choose $\sigma$ to be an outermost such arc on $D^{*}$. As $\left|D^{*} \cap K\right|=1$ we can choose $\sigma$ so that it cuts off a disk $E$ in $D^{*}$ that is disjoint from $K$. The boundary of $E$ consists of $\sigma$ and an arc $\alpha \subset Q$. The disk $E$ can be used to guide an isotopy of $Q$ isotoping a small neighborhood of $\alpha$ in $Q$ across $\Lambda$. This decreases $\left|D^{*} \cap \Lambda\right|$ contradicting our choice of $D^{*}$.

Claim 3. It suffices to consider the case that every component of $Q \cap \Lambda$ is either a circle parallel to the core of $\Lambda$ or is adjacent to $\gamma$.

Suppose $Q \cap \Lambda$ contains either an inessential circle in $\Lambda$ or an arc with both endpoints of $K$. In either case assume that this curve is disjoint from $\gamma$. Pick $\delta$ to 
be either an innermost such circle or an outermost such arc and let $G$ be the disk in $\Lambda$ that $\delta$ bounds. By minimality of $Q \cap \Lambda$ if $\delta$ is a circle, $G$ is a compressing disk for $Q$. If $\delta$ is an arc, then $G$ is a bridge disk and so there is a compressing disk for $Q$ contained in the boundary of a regular neighborhood of $G$. In either case we have found a compressing disk for $Q$ contained in $A_{K}$ and disjoint from $D^{*}$. If this disk is contained in $X$, then $Q_{K}$ is c-strongly compressible as the disk is disjoint from $D^{*}$. If the disk is contained in $Y$, the result follows by Case 1 .

By Claims 1 and 2 we may assume that $D^{*} \cap \Lambda=\gamma$ and by Claim 3 we may assume that every component of $Q \cap \Lambda$ is either a circle parallel to the core of $\Lambda$ or is adjacent to $\gamma$. Let $\sigma$ be the arc of $Q \cap \Lambda$ that is adjacent to $\gamma$. There are three cases to consider:

Subcase A: $\sigma$ is circle parallel to the core of $\Lambda$. As $Q \cap K \neq \varnothing$, there must be some $\operatorname{arc} \delta$ of $Q \cap \Lambda$ with both endpoints in $K$. But then $\delta$ is an $\operatorname{arc}$ of $Q \cap \Lambda$ that is disjoint from $\gamma$ contradicting our assumption.

Subcase B: $\sigma$ is a circle that is inessential in $\Lambda$. Let $E$ be the disk in $\Lambda$ that $\sigma$ bounds. By our assumptions the interior of $E$ is disjoint from $D^{*}$ and also from $Q$. Then $E$ is a compressing disk for $Q_{K}$ lying in $X$ that intersects $D^{*}$ in exactly one point. By Theorem 2.1 of [Tomova 2006] $Q_{K}$ is c-strongly compressible.

Subcase $C: \sigma$ is an arc:

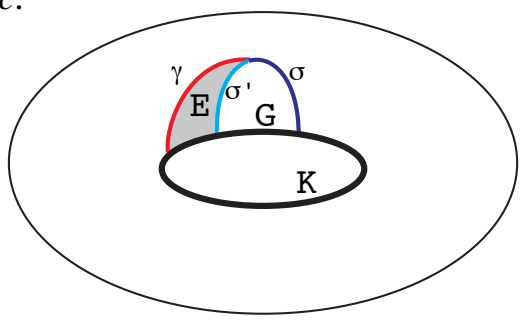

Let $G$ be the disk that $\sigma$ cuts from $\Lambda$. As before the boundary of a regular neighborhood of $G$ contains a compressing disk for $Q$ in $A_{K}$. If this disk lies in $Y$, Case 1 can be applied so we may assume $G$ lies in $X$. Thus the arc $\gamma$ lies outside of $G$. Let $E$ be the disk cobounded by $\gamma$, a subarc $\sigma^{\prime}$ of $\sigma$ and a subarc of $K$. Then the boundary of a regular neighborhood of $D^{*} \cup E$ contains a cut disk parallel to $D^{*}$ and a disk $F \subset Y$ :

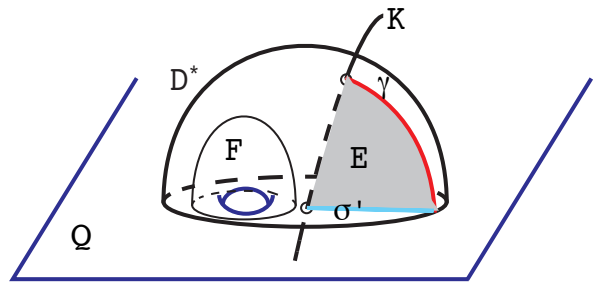


If $\partial F$ bounds a disk in $Q$, then $\partial D^{*}$ bounds a once punctured disk contradicting the fact that $D^{*}$ is a cut disk. If $\partial F$ bounds a punctured disk $D^{\prime}$ in $Q$, the $F \cup D^{\prime}$ is a sphere that intersects the knot once, a contradiction. Thus $\partial F$ is essential in $Q_{K}$ and so $F \subset Y$ is a compressing disk for $Q_{K}$ in $A_{K}$; therefore we can apply Case 1.

Finally, the following easy proposition will be used repeatedly in the proofs to follow.

Proposition 3.5. Suppose $P$ is a Heegaard surface for $M_{K}=A_{K} \cup_{P} B$ with genus at least 2 and $D^{c}$ is a cut disk for $P$ in $A_{K}$. Then there is a compressing disk $D$ for $P$ in $A_{K}$ such that $d\left(\partial D^{c}, \partial D\right)=1$.

Proof. Consider cut-compressing $A_{K}$ along $D^{c}$, i.e., remove a small open neighborhood of $D^{c}$ from $A_{K}$. The resulting 3-manifold is a $K$-handlebody containing a single bridge - the result of cutting the closed loop $K$. As $g(P) \geq 2$, the $K$ handlebody has genus at least 1 and thus there is a compressing disk $D$ which can be taken to be disjoint from the bridge. This disk is the desired compressing disk for $P$ in $A_{K}$.

\section{Configurations of $P$ and $Q$}

Let $K$ be any knot in any manifold $M$ except the unknot in $S^{3}$ and consider how $P$ (a Heegaard surface of $M_{K}=A_{K} \cup_{P} B$ ) and $Q$ (a Heegaard surface for $M=X \cup_{Q} Y$ that is also a bridge surface for $K$ ) intersect in $M$. Let $Q^{A}=Q \cap A, Q^{B}=Q \cap B$, $P^{X}=P \cap X$ and $P^{Y}=P \cap Y$ and let $\mathscr{A}$ and $\mathscr{B}$ be the collections of curves in $P$ that bound compressing disks in $A$ and $B$ respectively.

Definition 4.1. Suppose $S$ and $T$ are two properly embedded surfaces in a 3manifold $M$ containing a knot $K$ and assume $S$ and $T$ intersect the knot transversely. Let $c \in S_{K} \cap T_{K}$ be a simple closed curve bounding possibly punctured disks $D^{*} \subset S_{K}$ and $E^{*} \subset T_{K}$. If $D^{*}$ intersects $T_{K}$ only in curves that are inessential in $T_{K}$ and $E^{*}$ intersects $S_{K}$ only in curves that are inessential in $S_{K}$ we say that $c$ is removable.

Remark 4.2. Consider the curves of intersection between $P$ and $Q_{K}$.

(1) All removable curves can be removed via an isotopy of the surfaces that does not affect any essential curves of intersection.

(2) If all curves of intersection between $P$ and $Q_{K}$ are either essential in both surfaces or inessential in both surfaces then all inessential curves are removable.

Isotope $P$ and $Q$ so as to remove all removable curves of intersection. We will associate to a position of $P$ and $Q$ one or more of the following labels. 
- Label $A$ (resp. $B$ ) if some component of $Q_{K} \cap P$ is the boundary of a compressing disk for $P$ lying in $A_{K}$ (resp. $B$ ).

- Label $A^{c}$ if some component of $Q_{K} \cap P$ is the boundary of a cut disk for $P$ lying in $A_{K}$. (As $B \cap K=\varnothing$, no label $B^{c}$ can occur).

- Label $X$ (resp. $Y$ ) if there is a compressing disk for $Q_{K}$ lying in $X_{K}$ (resp. $Y_{K}$ ) that is disjoint from $P$ and the configuration does not already have labels $A, A^{c}$ or $B$.

- $X^{c}$ (resp. $Y^{c}$ ) if there is a cut disk for $Q_{K}$ lying in $X_{K}$ (resp. $Y_{K}$ ) that is disjoint from $P$ and the configuration does not already have labels $A, A^{c}$ or $B$.

- $x$ (resp. $y$ ) if some spine $\Sigma_{A_{K}}$ or $\Sigma_{B}$ lies entirely in $Y_{K}$ (resp. $X_{K}$ ) and the configuration does not already have labels $A, A^{c}$ or $B$.

We will use the superscript ${ }^{*}$ to denote the possible presence of superscript ${ }^{c}$, for example we will use $A^{*}$ if there is a label $A, A^{c}$ or both.

Lemma 4.3. If a configuration of $P$ and $Q$ has no labels, then $d(P) \leq 2-\chi\left(Q_{K}\right)$.

Proof. Suppose a configuration has no labels. First note that this implies $P \cap Q_{K} \neq$ $\varnothing$ as if $P$ is entirely contained in $X$ say, then the region would have a label $y$. If there is a curve of $P \cap Q_{K}$ that is inessential in $Q_{K}$ but essential in $P$ an innermost such curve in $Q_{K}$ would give rise to a label $A^{*}$ or $B$. If there is a curve of $P \cap Q_{K}$ that is inessential in $P$ but essential in $Q_{K}$ an innermost such curve in $P$ would give rise to a label $X^{*}$ or $Y^{*}$. Thus all curves of $P \cap Q_{K}$ are either essential in both surfaces or inessential in both. By Remark 4.2 we may assume all curves of $P \cap Q_{K}$ are essential in both surfaces. Furthermore, as no labels $X^{*}$ or $Y^{*}$ are associated to this configuration, $Q_{K}^{A}$ and $Q^{B}$ are both c-incompressible and as there are no labels $x$ or $y$, they each have at least one component that is not parallel to $P$. By Proposition 3.1 both $Q_{K}^{A}$ and $Q^{B}$ satisfy the hypothesis of Lemma 3.3 from which we deduce that $d(P) \leq 2-\chi\left(Q_{K}\right)$.

Lemma 4.4. If a configuration of $P$ and $Q$ has labels $A^{*}$ and $B$, then $d(P) \leq 2$.

Proof. If a configuration has labels $A$ and $B$, there are curves of $P \cap Q_{K}$, both essential in $P$, that bound compressing disks for $P$ in $A_{K}$ and $B$. As $P$ and $Q_{K}$ are embedded surfaces, these two curves are disjoint and thus $d(P) \leq 1$. If a configuration has labels $A^{c}$ and $B$, using the triangle inequality and Proposition 3.5 , we deduce that $d(P) \leq 2$.

Lemma 4.5. If a configuration has labels $x$ and $Y^{*}$, we are in at least one of the following situations: $Q_{K}$ is c-strongly compressible; $K$ is removable with respect to $Q ;$ ord $(P) \leq 2-\chi\left(Q_{K}\right)$. 
Proof. Assume $Q_{K}$ is c-weakly incompressible and $K$ is not removable. We will show that $d(P) \leq 2-\chi\left(Q_{K}\right)$. From the label $x$ we may assume, with no loss of generality, that there exists a spine for $A_{K}$ or $B$ contained in $Y_{K}$. Note that the spine of $A_{K}$ contains the frontier of an open neighborhood of $K$ and $K \cap Q \neq \varnothing$ thus we conclude that $\Sigma_{B} \subset Y_{K}$. From the label $Y^{*}$ we know that $Q_{K}$ has a c-disk in $Y_{K}-P$, call this disk $E$. By Lemma 3.4, $E \cap \Sigma_{B} \neq \varnothing$ so in particular $E \subset B$. Note that this also implies $Q_{K} \cap P \neq \varnothing$ as $Y \cap A \neq \varnothing$.

As no label $A^{*}$ or $B$ is present, any curve of intersection that is essential in $P$ must also be essential in $Q_{K}$. Suppose there is a curve that is essential in $Q_{K}$ and inessential in $P$. Let $D$ be the disk in $P$ an innermost such curve bounds. By Lemma 3.4 the disk $D$ cannot be in $Y_{K}$. If $D$ is in $X_{K}$, then it is disjoint from $E$ giving a c-strong compression for $Q_{K}$ contrary to our assumption. Thus we may assume that all curve of intersection are essential in both $P$ and $Q_{K}$.

Consider first $Q_{K}^{A}$. It is incompressible in $A_{K}$ because a compression into $Y_{K}$ would violate Lemma 3.4 and a compression into $X_{K}$ would provide a c-weak compression of $Q_{K}$. If $Q_{K}^{A}$ is not essential in $A_{K}$ then every component of $Q_{K}^{A}$ is parallel into $P$ so in particular $Q^{A} \cap K=\varnothing$. As it is always the case that $Q^{B} \cap K=\varnothing$ this implies that $Q \cap K=\varnothing$. As $Q$ is a bridge surface for $K$ that is not possible. We conclude that $Q_{K}^{A}$ is essential in $A_{K}$ so by Proposition 3.1 for each component $q$ of $Q_{K} \cap P$ that is not the boundary of a $P$-parallel annulus in $A_{K}$, the inequality $d(q, \mathscr{A}) \leq 1-\chi\left(Q_{K}^{A}\right)$ holds, i.e., $Q_{K}^{A}$ satisfies the hypotheses of Lemma 3.3.

By Lemma 3.4 $Q^{B}$ does not have compressing disks in $Y_{K} \cap\left(B-\Sigma_{B}\right)$ so it either has no compressing disks in $B-\Sigma_{B}$ at all or has a compressing disk lying in $X_{K}$. If $Q_{B}$ is incompressible in $B-\Sigma_{B} \cong P \times I$, then either it satisfies the hypothesis of Proposition 3.1 or each component of $Q_{B}$ is parallel to a subset of $P$. In the latter case the compressing disk $E$ of $Q^{B}$ in $Y_{K}-P$ can be extended via this parallelism to give a compressing disk for $P$ that is disjoint from all $q \in Q_{K} \cap P$. Hence

$$
d(q, \mathscr{B}) \leq 2 \leq 1-\chi\left(Q^{B}\right)
$$

as long as $Q^{B}$ is not a collection of $P$-parallel annuli. If that is the case, then $d\left(\partial E, q_{0}\right)=0$ for at least one $q_{0} \in\left(P \cap Q_{K}\right)$ so $d\left(q_{0}, \mathscr{B}\right) \leq 1 \leq 1-\chi\left(Q^{B}\right)$ as desired. Thus in this case $Q^{B}$ satisfies the hypothesis of Lemma 3.3 and we obtained the desired distance bound.

If $Q^{B}$ is bicompressible in $B$, then $Q^{B}$ is weakly incompressible as every compressing disk for $Q^{B}$ is also a compressing disk for $Q$. Because every compressing disk for $Q^{B}$ in $Y_{K}$ intersects $\Sigma_{B}, Q^{B}$ cannot be a tube-spanned recessed collar. By Theorem 3.2 $Q^{B}$ again satisfies the hypothesis of Lemma 3.3 and we can deduce the desired distance bound. 
Lemma 4.6. If a configuration has labels $X^{*}$ and $Y^{*}$, we are in at least one of the following situations: $Q_{K}$ is c-strongly compressible; $K$ is removable with respect to $Q ;$ ord $(P) \leq 2-\chi\left(Q_{K}\right)$.

Proof. By the labelling we can conclude that $Q_{K}$ has c-disks in both $X$ and $Y$ that are disjoint from $P$. Again we will assume that $Q_{K}$ is c-weakly incompressible and $K$ is not removable and show that the distance bound must hold. As before we may assume that all curves of $P \cap Q_{K}$ are essential in both surfaces. A curve that is essential in $P$ but inessential in $Q_{K}$ would lead to a label $A^{*}$ or $B$; a curve that is inessential in $P$ but essential in $Q_{K}$ would lead to c-strong compression of $Q_{K}$ as $Q_{K}$ has c-disks in both $X$ and $Y$ with boundaries disjoint from $P$. Thus all curves are either essential or inessential in both surfaces and all curves of intersection inessential in both surfaces have already been removed.

The c-disks for $Q_{K}$ giving rise to the labels $X^{*}$ and $Y^{*}$ must both be contained in $A_{K}$ or $B$ by c-weak incompressibility of $Q_{K}$. Suppose $Q_{K}^{A}$ is bicompressible and $Q_{B}$ is incompressible, the other case is similar. We may assume that $Q_{B}$ is essential as otherwise a label $x$ or $y$ would be present and Lemma 4.5 would apply. Thus $Q^{B}$ satisfies the hypothesis of Lemma 3.3. On the other hand $Q_{K}^{A}$ is a bicompressible c-weakly incompressible surface in a 3-manifold. By Theorem 3.2, $Q_{K}^{A}$ satisfies the hypothesis of Lemma 3.3 unless $Q_{K}^{A}$ has one component that is a tube spanned recessed collar and all other components are boundary parallel. In this case $Q_{K}^{A}$ would be disjoint from a spine of $A_{K}$ and thus the region would have a small label. The result then follows by Lemma 4.5.

Lemma 4.7. If a configuration has labels $x$ and $y$, we are in at least one of the following situations: $Q_{K}$ is c-strongly compressible; $K$ is removable with respect to $Q ;$ ord $(P) \leq 2-\chi\left(Q_{K}\right)$.

Proof. Again assume that $Q_{K}$ is c-weakly incompressible and $K$ is not removable. We can also assume that the configuration does not have any capital labels as otherwise we can apply Lemma 4.5. We may assume that all curves of intersection are essential in both $P$ and $Q_{K}$ for a curve that is essential in $P$ and inessential in $Q_{K}$ would give rise to a label $A^{*}$ or $B$ and a curve that is essential in $Q_{K}$ and inessential in $P$ would give rise to a label $X^{*}$ or $Y^{*}$. Moreover, as labels $X^{*}$ or $Y^{*}$ don't occur, $Q_{K}^{A}$ and $Q^{B}$ are c-incompressible in $M_{K}-P$.

Every spine of $A_{K}$ intersects $Q$ so both labels $x$ and $y$ must be due to a spine of $B$. Supposed there is a spine $\Sigma_{B} \subset Y_{K}$ and a spine $\Sigma_{B}^{\prime} \subset X_{K}$. Let $Q_{0}$ be a component of $Q^{B}$ that lies between the two spines. This implies that $Q_{0}$ is parallel into $P$ on both its sides, i.e., that $B \cong Q_{0} \times I$. As $g(P) \geq 2, Q_{0}$ is not an annulus.

Let $\alpha$ be an essential arc in $Q_{0}$ with endpoints in $P$. Then $\alpha \times I \subset Q_{0} \times I \cong B$ is a meridian disk $D$ for $B$ that intersects $Q_{0}$ precisely in $\alpha$. Consider the frontier of a regular neighborhood of $\left(Q_{0} \cup D\right) \cap P$. As $g(P) \geq 2$ the frontier contains 
at least one curve $\sigma$ that is essential in $P$. We can conclude that for every curve $q \in B \cap Q_{K}, d(\mathscr{B}, q) \leq d(\mathscr{B}, \sigma)+d(\sigma, q) \leq 2 \leq 1-\chi\left(Q^{B}\right)$. Thus $Q^{B}$ always satisfied the hypothesis of Lemma 3.3.

As we already saw $Q_{K}^{A}$ is essential so by Proposition $3.1 Q_{K}^{A}$ also satisfies the hypothesis of Lemma 3.3 and we are done by that lemma.

Let A represent any subset of the labels $A, A^{c}$, B the label $B$, X any subset of the labels $X, X^{c}, x$ and $\mathrm{Y}$ any subset of the labels $Y, Y^{c}, y$. Then the lemmas in this section can be summarized as follows.

Theorem 4.8. Suppose $Q_{K}$ is a c-weakly incompressible bridge surface for a knot $K$ such that $K$ is not removable with respect to $Q$ and $P$ is a Heegaard surface for the knot exterior. Let labels $\mathrm{A}, \mathrm{B}, \mathrm{X}$ and $\mathrm{Y}$ be defined as above. Then either every configuration of $P$ and $Q_{K}$ has exactly one of the labels $\mathrm{A}, \mathrm{B}, \mathrm{X}$ and $\mathrm{Y}$ associated to it or $d(P) \leq 2-\chi\left(Q_{K}\right)$.

Note that if $K$ is the unknot in $S^{3}$, then $P$ is a Heegaard splitting of the solid torus of genus at least 2 . As Heegaard splittings of a handlebody are standard, $P$ must be stabilized and thus $d(P)=0$.

\section{Two-parameter sweep-outs and their graphics}

Let $\Sigma_{(X, K)}$ and $\Sigma_{(Y, K)}$ continue to denote the spines for the $K$-handlebodies $X$ and $Y$. Then there is a map $H:(Q, Q \cap K) \times I \rightarrow(M, K)$ that is a homeomorphism except over $\Sigma_{(X, K)} \cup \Sigma_{(Y, K)}$ and, near $Q \times \partial I$, the map $H$ gives a mapping cylinder structure to a neighborhood of $\Sigma_{(X, K)} \cup \Sigma_{(Y, K)}$. Little is lost and some brevity gained if we restrict $H$ to $Q_{K} \times(I, \partial I) \rightarrow\left(M_{K}, \Sigma_{(X, K)} \cup \Sigma_{(Y, K)}\right)$. The map $H$ is then called a sweep-out associated to $Q$. Similarly there is a sweep-out associated to the Heegaard surface $P$ between the spines $\Sigma_{A_{K}}$ and $\Sigma_{B}$.

Consider simultaneous sweep-outs of $P$, between $\Sigma_{A_{K}}$ and $\Sigma_{B}$ and of $Q_{K}$ between $\Sigma_{(X, K)}$ and $\Sigma_{(Y, K)}$. This two-parameter sweep-out can be described by a square where each point in the interior of the square represents a position of $P$ and $Q_{K}$. Inside the square is a graphic $\Gamma$ which represents all points where the intersection of the two surfaces is not generic. At each edge of $\Gamma$ there is a single tangency between $P$ and $Q_{K}$ and at each valence 4 vertex there are two tangencies. Each component of the complement of $\Gamma$ will be called a region and to each region we can associate labels as defined in Section 4. Two regions are adjacent if they share an edge. For the moment we will restrict our attention to the case when $Q_{K}$ is a c-weakly incompressible surface and $K$ is not removable with respect to $Q$, thus by Theorem 4.8 we may assume that either $d(P) \leq 2-\chi\left(Q_{K}\right)$ or every region has exactly one of the labels $\mathrm{A}, \mathrm{B}, \mathrm{X}$ and $\mathrm{Y}$. In the former case we have achieved the desired distance bound. 
Lemma 5.1. If two adjacent regions are labelled $\mathrm{A}$ and $\mathrm{B}$, then $d(P) \leq 2$.

Proof. As the two regions are adjacent, we can transform the configuration caring the labels $\mathrm{A}$ into the configuration caring the label в by isotoping $P$ through a single tangency of $Q_{K}$. Notice that the curves of intersection of $P$ and $Q_{K}$ before the isotopy are disjoint from the curves of intersection after the isotopy. Thus there is a c-disk for $A_{K}, D_{A}^{*}$ and a compressing disk for $B, D_{B}$ such that $D_{A}^{*} \cap D_{B}=\varnothing$. If $D_{A}^{*}$ is actually a compressing disk, that would imply that $d(P) \leq 1$. If $D_{A}^{*}$ is a cut disk, then we apply Proposition 3.5 and the triangle inequality to conclude that $d(P) \leq 2$.

Lemma 5.2. Suppose a configuration is changed by passing through a saddle point, and the bigon $C$ defining the saddle tangency lies in $X_{K} \cap A_{K}$ (The case when the bigon lies in the handlebody $B$ is similar). Then

- No label $x$ or $X^{*}$ is removed.

- No label y or $Y^{*}$ is created.

- If there is no label $x$ or $X^{*}$ before the move, but one is created after and if there is a label $y$ or $Y^{*}$ before the move and none after, then $d(P) \leq 2-\chi\left(Q_{K}\right)$.

Proof. Much of the argument here parallels the argument in the proof of Lemma 4.1 in [Scharlemann and Tomova 2006a].

We first show that no label $x$ or $X^{*}$ is removed. If there is a c-disk for $X_{K}$ that lies in $A_{K}$, a standard innermost disk, outermost arc argument on its intersection with $C$ shows that there is a c-disk for $X_{K}$ in $A_{K}$ that is disjoint from $C$. The saddle move has no effect on such a disk (nor, clearly, on a c-disk for $X_{K}$ that lies in $B$ ). If there is a spine of $A_{K}$ or of $B$ lying entirely in $Y_{K}$ then that spine, too, is unaffected by the saddle move.

Dually, no label $y$ or $Y^{*}$ is created: the inverse saddle move, restoring the original configuration, is via a bigon that lies in $B \cap Y_{K}$.

To prove the third item position $Q_{K}$ so that it is exactly tangent to $P$ at the saddle. A bicollar of $Q_{K}$ then has ends that correspond to the position of $Q_{K}$ just before the move and just after. Let $Q_{K}^{a}$ denote $Q_{K} \cap A_{K}$ after the move and $Q_{K}^{b}$ denote $Q_{K} \cap B$ before the move. The bicollar description shows that $Q_{K}^{a}$ and $Q_{K}^{b}$ have disjoint boundaries in $P$. Moreover the complement of $Q_{K}^{a} \cup Q_{K}^{b}$ in $Q_{K}$ is a regular neighborhood of the singular component of $P \cap Q_{K}$, with Euler characteristic -1 . It follows that $\chi\left(Q_{K}^{a}\right)+\chi\left(Q_{K}^{b}\right)=\chi\left(Q_{K}\right)+1$.

With $Q_{K}$ positioned as described, tangent to $P$ at the saddle point but otherwise in general position, consider the closed (nonsingular) curves of intersection.

Claim 1. It suffices to consider the case in which all nonsingular curves of intersection are essential in $P$. 
First note that any curve of intersection that is inessential in $Q_{K}$ must be inessential in $P$ as no labels $A^{*}$ or $B$ are present. To prove the claim, suppose a nonsingular curve is inessential in $P$ and consider an innermost one. Assume first that the disk $D$ that it bounds in $P$ does not contain the singular curve $s$. If $\partial D$ is essential in $Q_{K}$, then it would give rise to a label $X$ or a label $Y$ that persists from before the move until after the move, contradicting the hypothesis. Thus any inessential curve in $P$ that doesn't bound a disk containing the singular curve $s$ is also inessential in $Q_{K}$ and can be removed without affecting the label of the region.

Suppose then that the disk $D \subset P$ contains the singular component $s$. By the above, $s$ is the only component of $Q_{K} \cap P$ in the interior of $D$. When the saddle is pushed through, the number of components in $s$ switches from one, $s_{0}$, to two, $s_{ \pm}$, or vice versa. All three curves are inessential in $P$ since they lie in $D$. The curve $s_{0}$ and at least one of $s_{ \pm}$bound disks in $P$ whose interiors are disjoint from $Q_{K}$. If one of these curves was essential in $Q_{K}$ that would give rise to a label $X$ or $Y$ that persists through the isotopy. As no such label exists, both of these curves are inessential in $Q_{K}$, i.e., bound a possibly one-punctured disks in $Q_{K}$. As no sphere in $M$ can intersect $K$ exactly once, $s_{0}$ and one of $s_{ \pm}$bound disks in $Q_{K}$. Because the three curve cobound a pair of pants in $Q_{K}$, all three curves $s_{0}$ and $s_{ \pm}$ are inessential in $Q_{K}$. This means that all three curves are removable so passing through this saddle cannot have an effect on the labelling.

Claim 2. It suffices to consider the case in which also all three curves $s_{0}, s_{ \pm}$are essential in $P$.

The case in which all three curves are inessential in $P$ is covered in the proof of Claim 1. If two are inessential in $P$ so is the third. Thus the only remaining case is that exactly one of the curves $s_{0}, s_{ \pm}$is inessential in $P$ and by Claim 1, the disk it bounds in $P$ is disjoint from $Q$. As before the curve cannot be essential in $Q_{K}$ as otherwise it will give rise to a label $X$ or $Y$ that persists through the isotopy. Thus the curve is inessential in $Q_{K}$ also (in fact it must bound a disk there) so it is removable. If this curve is $s_{ \pm}$, passing through the saddle can have no effect on the labelling. If the removable curve is $s_{0}$, then the curves $s_{ \pm}$are parallel in both surfaces. Passing through the saddle has the same effect on the labelling as passing an annulus component of $P^{X}$ across a parallel annulus component $Q^{0}$ of $Q_{K}^{A}$. This move can have no effect on labels $x$ or $y$. As there is a label $Y^{*}$ before the move, there is a meridian, possibly punctured disk $E^{*}$ for $Y_{K}$ that is disjoint from $P$. This disk would persist after the move, unless $\partial E^{*}$ is in fact the core curve of the annulus $Q^{0}$. But then the union of $E^{*}$ and half of $Q^{0}$ would be a possibly punctured meridian disk of $A_{K}$ bounded by a component of $\partial Q^{0} \subset P$. In other words, there would have to have been a label $A^{*}$ before the move, a final contradiction establishing Claim 2. 
The above two claims allow us to assume that all curves of intersection before and after the move are essential in both surfaces. Note that $Q_{K}^{a}$ and $Q_{K}^{b}$ are cincompressible (as there are no labels $X^{*}$ or $Y^{*}$ persisting through the move) and have at least one component that is not parallel to $P$ (as there are no labels $x$ or $y$ persisting through the move). Now apply Proposition 3.1 to both sides: Let $q_{a}$ (resp. $q_{b}$ ) be a boundary component of an essential component of $Q_{K}^{a}$ (resp. $Q_{K}^{b}$ ). Then

$$
\begin{aligned}
d(P) & =d(\mathscr{A}, \mathscr{B}) \leq d\left(q_{a}, \mathscr{A}\right)+d\left(q_{a}, q_{b}\right)+d\left(q_{b}, \mathscr{B}\right) \\
& \leq 3-\chi\left(Q_{K}^{a}\right)-\chi\left(Q_{K}^{b}\right)=2-\chi\left(Q_{K}\right)
\end{aligned}
$$

as required.

Lemma 5.3. If some vertex of $\Gamma$ is surrounded by regions labelled with all four labels $\mathrm{A}, \mathrm{B}, \mathrm{X}$ and $\mathrm{Y}$, we are in at least one of the following situations: $Q_{K}$ is c-strongly compressible; $K$ is removable with respect to $Q ;$ or $d(P) \leq 2-\chi\left(Q_{K}\right)$.

Proof. Suppose there is such a vertex of $\Gamma$ and assume that $Q_{K}$ is c-weakly incompressible and $K$ is not removable with respect to $Q$. As we have already established the desired distance bound if any region has more than one label or if two adjacent regions are labelled $\mathrm{A}$ and $\mathrm{B}$ or $\mathrm{X}$ and $\mathrm{Y}$ we may assume that going around the vertex the regions are labelled in the order A, X, B and Y. Note then that only two saddle moves are needed to move from a configuration labelled $A^{*}$ to one labelled $B$. The former configuration includes a c-disk for $P$ in $A_{K}$ and the latter a compressing disk for $P$ in $B$. Recall that all curves of intersection before a saddle move are disjoint from all curves of intersection after the saddle move. Using Proposition 3.5 and the triangle inequality, it follows that $d(K, P) \leq 3 \leq 2-\chi\left(Q_{K}\right)$, as long as at least one of the regions labelled $\mathrm{X}$ and $\mathrm{Y}$ contains at least one essential curve.

Suppose all curves of $P \cap Q_{K}$ in the regions with labels $\mathrm{X}$ and $\mathrm{Y}$ are inessential. Consider the region labelled $\mathrm{X}$. Crossing the edge in the graphic from this region to the region labelled A corresponds to attaching a band $b_{A}$ with both endpoints in an inessential curve $c \in P \cap Q$. Note that attaching this band must produce an essential curve that gives rise to the label $\mathrm{A}$, call this curve $c_{A}$. Similarly crossing the edge from the region $\mathrm{X}$ into the region $B$ corresponds to attaching a band $b_{B}$ to give a curve $c_{B}$. The two bands must be attached to the same curve $c$ otherwise $c_{A}$ and $c_{B}$ would be disjoint curves giving rise to labels $\mathrm{A}$ and $\mathrm{B}$. As we assumed that in the region labelled $\mathrm{Y}$ all curves of intersection are inessential, attaching both bands simultaneously results in an inessential curve $c_{A B}$. But that can only occur of $P$ is a torus which we know not to be the case.

Lemma 5.4. If a label $\mathrm{B}$ appears in the regions adjacent to the $\Sigma_{A_{K}}$ side of $I \times I$, or a label $\mathrm{A}$ appears along the $\Sigma_{B}$ side, or a label $\mathrm{Y}$ appears along the $\Sigma_{(X, K)}$ side, or a label $\mathrm{X}$ appears along the $\Sigma_{(Y, K)}$ side, we are in at least one of the following 
situations: $Q_{K}$ is c-strongly compressible; $K$ is removable with respect to $Q$; or $d(P) \leq 2-\chi\left(Q_{K}\right)$.

Proof. Suppose $Q_{K}$ is a c-weakly incompressible surface and $K$ is not removable with respect to $Q$. Consider first a region that is adjacent to the $\Sigma_{A_{K}}$ side of $I \times I$. In such a region $P$ is the boundary of a small neighborhood of $\Sigma_{A_{K}}$ and $Q$ either intersects it in meridional circles or doesn't intersect it at all. In the former case any curve of intersection $P \cap Q_{K}$ would lead to a label A so if a label B also appears, then $d(P) \leq 2$ by Lemma 4.4. In the latter case no label $\mathrm{B}$, or in fact label $\mathrm{A}$, is possible. Similarly if $P$ is near $\Sigma_{B}$ and the configuration carries a label A then $d(P) \leq 2$.

Suppose now that $Q_{K}$ is near $\Sigma_{(X, K)}$. As $P$ intersects $\Sigma_{(X, K)}$ transversely, all but a finite number of points of $\Sigma_{(X, K)}$ will be disjoint from $P$. Thus a label $\mathrm{X}$ necessarily occurs. If a label Y also occurs, then $d(P) \leq 2-\chi\left(Q_{K}\right)$ by Lemma 4.6. Symmetrically the distance bound holds if some region adjacent to the $\Sigma_{(Y, K)}$ boundary of the square is labelled $\mathrm{X}$.

Finally we will make use of the following combinatorial result.

Theorem 5.5. [Sperner's Lemma] Suppose a square I $\times I$ contains a graph $\Gamma$ such that all vertices of $\Gamma$ in the interior of the square are valence 4 and all vertices contained in the boundary of the square are valence 1 . Suppose each component of $I \times I-\Gamma$ is labelled with exactly one of the labels $N, E, S$, or $W$ in such a way that

(1) no region on the East side of $I \times I$ is labelled $W$, no region on the West side is labelled $E$, no region on the South side is labelled $N$ and no region on the North side is labelled $S$.

(2) no two adjacent regions are labelled $E$ and $W$ nor $N$ and $S$.

Then some valence 4 vertex is surrounded by regions carrying all 4 labels.

Theorem 5.6. Let $K$ be a knot in a closed irreducible 3-manifold $M$ and let $P$ be a Heegaard splitting of the knot complement such that $g(P) \geq 2$. Suppose $Q$ is a bridge surface for $K$. we are in at least one of the following situations: $Q_{K}$ is c-strongly compressible; $K$ is removable with respect to $Q ;$ or $d(P) \leq 2-\chi\left(Q_{K}\right)$.

Proof. Suppose that $Q_{K}$ is c-weakly incompressible and $K$ is not removable with respect to $Q$. Consider a 2-parameter sweep-out of $P$ and $Q_{K}$ and the associated graphic $\Gamma \in I \times I$. Label the regions of $I \times I-\Gamma$ with the labels $\mathrm{A}, \mathrm{B}, \mathrm{X}$ and $\mathrm{Y}$ as described before. By Theorem 4.8 we may assume that each region has a unique label. By Lemmas 5.1 and 5.2 we may assume that no two adjacent regions are labelled A and B or X and Y. Finally Lemma 5.4 shows that the labels of regions adjacent to the boundaries of $I \times I$ satisfy the conditions that no label B appears 
in the regions adjacent to the $\Sigma_{A_{K}}$ side of $I \times I$, no label A appears along the $\Sigma_{B}$ side, no label $\mathrm{Y}$ appears along the $\Sigma_{(X, K)}$ side, and no label $\mathrm{X}$ appears along the $\Sigma_{(Y, K)}$ side of the square. By Theorem 5.5 there is a valence 4 vertex of $\Gamma$ that is surrounded by regions carrying all four labels. The distance bound then follows from Lemma 5.3.

Now we can proof the main result in this paper. We recall the theorem for the convenience of the reader.

Theorem 1.3. Let $K$ be a knot in a closed oriented irreducible 3-manifold $M$ and let $P$ be a Heegaard splitting of the knot complement of genus at least two. Suppose $Q$ is a bridge surface for $K$. Then either

- $d(P) \leq 2-\chi(Q-N(K))$, or

- $K$ can be isotoped to be disjoint from $Q$ so that after the isotopy $Q$ is a Heegaard surface for $M_{K}$ that is isotopic to a possibly stabilized copy of $P$.

Proof. If $Q_{K}$ is stabilized, meridionally stabilized or perturbed, as described in Section 2, there is a bridge surface for $K, Q^{\prime}$, such that $\chi\left(Q_{K}^{\prime}\right) \geq \chi\left(Q_{K}\right)$. By possibly replacing $Q_{K}$ by $Q_{K}^{\prime}$ we may assume $Q_{K}$ is not stabilized, meridionally stabilized or perturbed. If $Q_{K}$ is removable, then again by the results in Section 2 there is a Heegaard surface $\tilde{Q}$ for $M_{K}$ that is isotopic to $Q$ in $M$. By hypothesis $\tilde{Q}$ is not isotopic to a possibly stabilized copy of $P$ so the result follows by Theorem 1.2. If $Q_{K}$ is c-weakly incompressible, the result follows by Theorem 5.6. The only remaining case is that $Q_{K}$ is a c-strongly compressible bridge surface for $K$ that is not stabilized, meridionally stabilized, perturbed or removable. By Theorem 2.1 in this case there is an essential surface $F$ with $\chi\left(F_{K}\right) \geq \chi\left(Q_{K}\right)$. Using Theorem 1.1 we deduce that $d(P) \leq 2-\chi(F) \leq 2-\chi\left(Q_{K}\right)$.

In the case when the manifold is $S^{3}$ we can also eliminate the restriction on the genus of $P$ by assuming that $K$ is nontrivial as that implies that the genus of any Heegaard splitting of $S_{K}^{3}$ is at least 2.

Corollary 5.7. Let $K$ be a nontrivial knot in $S^{3}$ and let $P$ be a Heegaard splitting of the knot complement. Suppose $Q$ is a bridge surface for $K$. Then either

- $d(P) \leq 2-\chi(Q-N(K))$, or

- $K$ can be isotoped to be disjoint from $Q$ so that after the isotopy $Q$ is a Heegaard surface for the knot exterior that is isotopic to a possibly stabilized copy of $P$.

Notice that if $K$ can be isotoped to be disjoint from $Q$ so that $Q$ is a Heegaard surface for $M_{K}$ that is isotopic to a possibly stabilized copy of $P$, then $g(Q) \geq$ $g(P)$. The following corollary then follows immediately from Theorem 1.3 if 
$g(Q) \geq 1$ and from Corollary 5.7 if $g(Q)=0$ as the only manifold with a genus 0 Heegaard splitting is $S^{3}$.

Corollary 5.8. Let $K$ be a knot in a closed irreducible 3-manifold $M$ (except the unknot in $\left.S^{3}\right)$ and let $P$ be a Heegaard splitting of the knot complement. Suppose $Q$ is a bridge surface for $K$ such that $g(Q)<g(P)$. Then $d(P) \leq 2-\chi(Q-N(K))$.

\title{
References
}

[Bachman and Schleimer 2005] D. Bachman and S. Schleimer, "Distance and bridge position", Pacific J. Math. 219:2 (2005), 221-235. MR 2007a:57028 Zbl 1086.57011

[Hartshorn 2002] K. Hartshorn, "Heegaard splittings of Haken manifolds have bounded distance", Pacific J. Math. 204:1 (2002), 61-75. MR 2003a:57037 Zbl 1065.57021

[Hayashi and Shimokawa 2001] C. Hayashi and K. Shimokawa, "Heegaard splittings of the pair of the solid torus and the core loop", Rev. Mat. Complut. 14:2 (2001), 479-501. MR 2003a:57038 Zbl 0996.57012

[Hempel 2001] J. Hempel, "3-manifolds as viewed from the curve complex", Topology 40:3 (2001), 631-657. MR 2002f:57044 Zbl 0985.57014

[Johnson and Thompson 2006] J. Johnson and A. Thompson, "On tunnel number one knots that are not $(1, n)$ ”, preprint, 2006. arXiv math.GT/0606226

[Kobayashi and Rieck 2007] T. Kobayashi and Y. Rieck, "Knot exteriors with additive Heegaard genus and Morimoto's conjecture", preprint, 2007. arXiv math.GT/0701765

[Minsky et al. 2006] Y. Minsky, Y. Moriah, and S. Schleimer, "High distance knots", preprint, 2006. arXiv math.GT/0607265

[Morimoto 2000] K. Morimoto, "On the super additivity of tunnel number of knots", Math. Ann. 317:3 (2000), 489-508. MR 2001g:57016 Zbl 0981.57005

[Scharlemann 2006] M. Scharlemann, "Proximity in the curve complex: boundary reduction and bicompressible surfaces", Pacific J. Math. 228:2 (2006), 325-348. MR 2008c:57035 Zbl 1127.57010

[Scharlemann and Tomova 2006a] M. Scharlemann and M. Tomova, "Alternate Heegaard genus bounds distance”, Geom. Topol. 10 (2006), 593-617. MR 2007b:57040 Zbl 1128.57022

[Scharlemann and Tomova 2006b] M. Scharlemann and M. Tomova, "Conway products and links with multiple bridge surfaces”, preprint, 2006. arXiv math.GT/0608435

[Scharlemann and Tomova 2006c] M. Scharlemann and M. Tomova, "Uniqueness of bridge surfaces for 2-bridge knots", preprint, 2006. arXiv math.GT/0609567

[Tomova 2006] M. Tomova, “Thin position for knots in a 3-manifold", preprint, 2006. arXiv math/ 0609674.

[Tomova 2007] M. Tomova, "Multiple bridge surfaces restrict knot distance", Algebr. Geom. Topol. 7 (2007), 957-1006. MR 2336246 Zbl 05220900

Received May 18, 2007.

\author{
MagGY TOMOVA \\ MATHEMATICS DEPARTMENT \\ RICE UNIVERSITY \\ HOUSTON, TX 77005 \\ UNITED STATES \\ maggy.tomova@rice.edu \\ http://www.owlnet.rice.edu/ mt2/
}

